\title{
Resilience in the mountains: biocultural refugia of wild food in the Greater Caucasus Range, Azerbaijan
}

\author{
Renata Sõukand $^{1 *}$ and Andrea Pieroni ${ }^{2}$
}

\footnotetext{
${ }^{1}$ Department of Environmental Sciences, Informatics, and Statistics, Ca' Foscari University of Venice, Via Torino 155, 30172 Mestre, Venice, Italy *renata.soukand@unive.it, +39-392-160-4529,+372-5022394 (corresponding author)

${ }^{2}$ University of Gastronomic Sciences, Piazza Vittorio Emanuele II 9, I-12060 Pollenzo, Cuneo, Italy
}

\begin{abstract}
Diversity of Traditional Ecological Knowledge (TEK) created on the edges of culture is the key to the sustainability and resilience of humankind. We recorded wild food TEK among seven autochthonous linguistic communities living on both sides of the Greater Caucasus Range, documenting the use of 72 wild taxa as well as remarkable diversity of both taxa and uses among the communities. The most isolated communities form distinct biocultural refugia for wild food plants and their uses, but the sustainability of such communities is under threat due to depopulation, and their TEK has already entered into decline. While isolation may have been responsible for the preservation of food biocultural refugia, it may no longer be enough for the passive preservation of the food refugia in the study area in the future. More proactive steps have to be taken in order to ensure the sustainability of TEK of the study communities and beyond.
\end{abstract}

Keywords: biocultural refugia, wild food plants, Azerbaijan, autochthonous languages of Caucasus, Traditional Ecological Knowledge, ethnic and linguistic minorities

\section{Introduction}

Diversity of Traditional Ecological Knowledge (TEK) is the key to the sustainability and resilience of humankind within changing climatic and socio-economic conditions. Cultural and linguistic edges create a great diversity of TEK, including that of wild food (Pieroni et al. 2018), yet there are many mechanisms that can limit diversity and erode TEK. Barthel et al. introduced the term biocultural refugia to refer to "sources of resilience in the landscape of food production" (2013a). The same authors later suggested that places holding biocultural refugia are important for policy makers and must be considered as part of the solutions addressing global change (Barthel et al. 2013b).

Recent research has shown that centralization has homogenized and eroded plant use in Post-Soviet countries. For example, regional differences in Ukraine are relatively minor despite the separation, distance and linguistic differences (Soukand and Pieroni 2016, Pieroni and Soukand 2017, 2018), in Central Belarus the use of wild food plants has decreased to multifunctional ones (Soukand et al. 2017a), and in the Latgale region of Latvia linguistic and religious differences did not save TEK from homogenization (Mezaka et al 2019 unpublished field results). At the same time, culturally undisturbed regions still hold considerable biocultural richness (Savo et al 2019). Also, quite surprisingly, results from the Republic of Georgia have highlighted the extremely large variety of plant uses in all spheres of life (Bussmann et al. 2016). The South Caucasus, of which Georgia is a part, served as a gateway to Northern Eurasia for the initial spread of animal and plant domestication about 12,000 years ago. In more recent times the region was part of the heavily centralized Soviet Union, the establishment and collapse of which brought food shortages and at the same time, due to forceful relocation of ethnic minorities and collectivization, a significant cut-off of traditional lifestyles. Therefore, this region offers interesting ground for research, as a few recent investigations in the region (Georgia (Bussmann et al., 2016, 2017, 2018; Łuczaj et al. 2017), Armenia (Hovsepyan et al. 2016), Azerbaijan (Pieroni and Soukand 2019) and Dagestan (Kaliszewska and Kołodziejska-Degórska 2015)) have demonstrated that the mountain villages still hold significant biocultural diversity and can be considered a potential biocultural refugia. While a comprehensive volume on the ethnobotany of the Caucasus has already been published (Bussmann 2017), it provides only an overview of the currently and historically most used taxa in the whole region and does not indicate the small-scale regional and ethnic divergences of plant use, due to its summary format. 
To date, only one autochthonous linguistic group in Azerbaijan (Udis) has been studied with regard to wild food plants (Pieroni and Soukand 2019), despite the fact that many of them could still hold a high diversity of TEK as they are relatively isolated and have been settled in the region for about a millennium. Azerbaijan has the lowest Global Food Security Index (2018) in Europe which could also signal the potential need to use and maintain the sustainability of wild food resources, as low income often implies more extensive use of wild foods (Stryamets et al. 2015). Therefore, we expect to see diversity of TEK in the region despite the long-lasting influence of the centralization and homogenization practiced during the height of the Soviet Union.

The aims of this study were a) to record the traditional plant foraging among seven autochthonous linguistic communities living on both sides of the Greater Caucasus Range, b) to compare the uses of wild food plants among the communities in order to identify possible differences and define food plant cultural markers (sensu Pieroni et al. 2015: plants used and mentioned exclusively by one cultural group), c) to discuss the influence of different factors (like linguistic and cultural distance, isolation and separation) on the resilience and sustainability of TEK, and d) to provide recommendations for strengthening the position of TEK in the study communities.

\section{Data and Methods}

Study area, communities and the field study

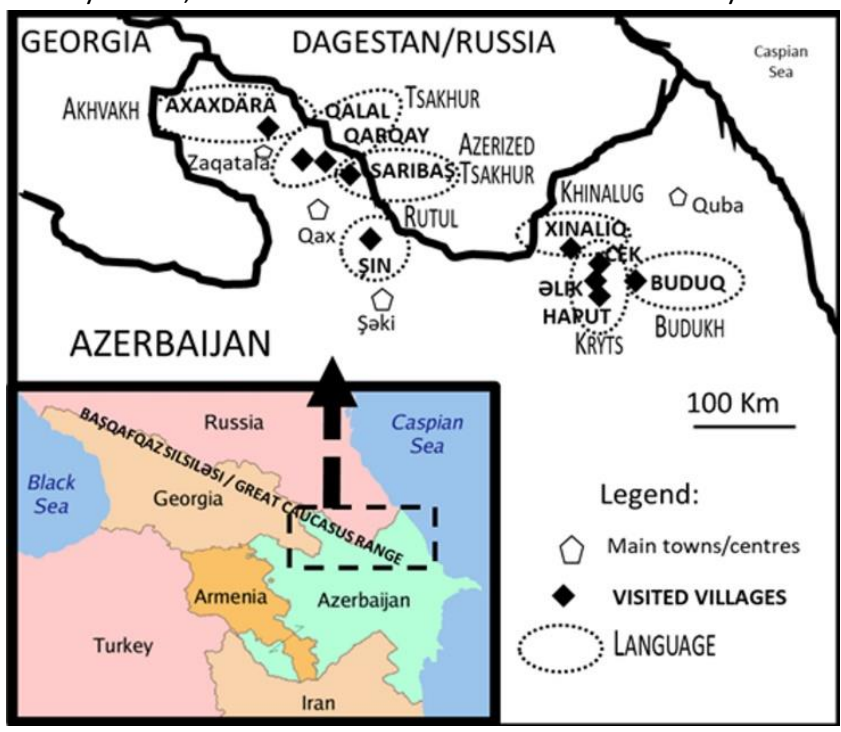

Ten villages inhabited by seven distinct linguistic groups were visited in November 2017 and October 2018. Visited villages (Figure 1) are situated on both sides of the Eastern Greater Caucasus Range. Of these, Budukh, Khinalug and Kryts communities are situated on the north-eastern side of the range in the Quba-Khachmaz region (hereafter "NW group"), while the other four, Akhvakh, Rutul and two Tsakhur communities, are located in the Shaki-Zaqatala region on the south-western side of the range (hereafter "SE group"). One of the Tsakhur communities has heavily adapted to the Azeri mainstream along the last century, and its members consider themselves Azeris and speak the Azeri language, although they also acknowledge their Tsakhur ancestry; and thus they are referred to as "azerized Tsakhurs".

Figure 1 . Study area and visited villages.

Both regions border Dagestan. The study communities are autochthonous and the visited villages are located in mountainous areas. All the communities are bilingual and elderly community members, especially those who served in the Soviet army or worked in Russia, speak Russian as a third language. The languages of the communities belong to the Northeast Caucasian language family, apart from the language spoken in Saribas, where the villagers are highly Azerized and speak Azerbaijani (which belongs to the Turkic language family) with some relics from Tsakhur languages. The communities remained endogamic until recent times and are (mainly Sunni) Muslim. The majority of the interviewees were middle-aged or elderly individuals identified by community members (mainly local farmers or shepherds) as knowledge holders; however, in almost all of the communities some younger people were also included in the sample. The villages are located within an altitudinal range of 700 to 2100 meters above sea level, yet the villages situated at lower altitudes have good access to the 
Sõukand, R. and Pieroni, A. 2019. Resilience in the mountains: biocultural refugia of wild food in the Greater Caucasus Range, Azerbaijan. Biodiversity and Conservation, 28 (13): 3529-3545.

higher mountains found in close proximity and within walking distance. Detailed characteristics of the study communities are presented in Table 1.

Table 1. Characteristics of the study communities and interviewed participants.

\begin{tabular}{|c|c|c|c|c|c|c|c|}
\hline $\begin{array}{l}\text { Ethnic or ethno- } \\
\text { religious group }\end{array}$ & Akhvakh & $\begin{array}{l}\text { "Azerized } \\
\text { Tsakhur" }\end{array}$ & Budukh & Khinalug & Kryts & Rutul & Tsakhur \\
\hline $\begin{array}{l}\text { Approx. number } \\
\text { of inhabitants in } \\
\text { Azerbaijan }\end{array}$ & 100 & 100 & $\begin{array}{l}1000 \quad(200 \\
\text { speak } \\
\text { language })\end{array}$ & 1000 & 5000 & 30000 & 10000 \\
\hline $\begin{array}{l}\text { Level of } \\
\text { isolation }\end{array}$ & $\begin{array}{l}\text { Slightly } \\
\text { isolated } \\
\text { (good road, } \\
\text { close to } \\
\text { bigger town) }\end{array}$ & $\begin{array}{l}\text { Relatively } \\
\text { isolated } \\
\text { (separated, } \\
\text { now relatively } \\
\text { good road) }\end{array}$ & $\begin{array}{l}\text { Highly } \\
\text { isolated (very } \\
\text { bad mountain } \\
\text { and river-bed } \\
\text { road) }\end{array}$ & $\begin{array}{l}\text { Relatively } \\
\text { isolated } \\
\text { (separated, } \\
\text { relatively } \\
\text { good road) }\end{array}$ & $\begin{array}{l}\text { Highly } \\
\text { isolated } \\
\text { (very bad } \\
\text { mountain } \\
\text { road) }\end{array}$ & $\begin{array}{l}\text { Slightly } \\
\text { isolated (good } \\
\text { road) }\end{array}$ & $\begin{array}{l}\text { Highly isolated } \\
\text { (very bad } \\
\text { mountain and } \\
\text { river-bed road) }\end{array}$ \\
\hline $\begin{array}{l}\text { Approximate } \\
\text { average elevation }\end{array}$ & $700 \mathrm{~m}$ & $1600 \mathrm{~m}$ & $1700 \mathrm{~m}$ & $2100 \mathrm{~m}$ & $1700 \mathrm{~m}$ & $1000 \mathrm{~m}$ & $1100 \mathrm{~m}$ \\
\hline $\begin{array}{l}\text { Original } \\
\text { language } \\
\text { (language } \\
\text { family) written } \\
\text { or not }\end{array}$ & $\begin{array}{l}\text { Akhvakh } \\
\text { (Avar-Andic, } \\
\text { Andic) } \\
\text { unwritten, } \\
\text { home use } \\
\text { only }\end{array}$ & $\begin{array}{l}\text { Azerbaijani } \\
\text { (Turkic, } \\
\text { Western } \\
\text { Oghuz), } \\
\text { village dialect }\end{array}$ & $\begin{array}{l}\text { Budukh, } \\
\text { (Lezgic, } \\
\text { Southern } \\
\text { Samur) } \\
\text { unwritten, all } \\
\text { domains }\end{array}$ & $\begin{array}{l}\text { Khinalug, } \\
\text { (Lezgic, } \\
\text { independent } \\
\text { branch) } \\
\text { unwritten, all } \\
\text { domains }\end{array}$ & $\begin{array}{l}\text { Kryts } \\
\text { (Lezgic, } \\
\text { Southern } \\
\text { Samur) } \\
\text { unwritten, } \\
\text { all domains }\end{array}$ & $\begin{array}{l}\text { Rutul (Lezgic, } \\
\text { Western } \\
\text { Samur) written } \\
\text { since the } \\
\text { 1990s, mainly } \\
\text { home use }\end{array}$ & $\begin{array}{l}\text { Tsakhur (Lezgic, } \\
\text { Western Samur) } \\
\text { written since the } \\
\text { 1930s, all } \\
\text { domains }\end{array}$ \\
\hline $\begin{array}{l}\text { Language spoken } \\
\text { in the studied } \\
\text { villages }\end{array}$ & $\begin{array}{l}\text { Akhvakh and } \\
\text { Russian or } \\
\text { Azeri }\end{array}$ & $\begin{array}{l}\text { Azerbaijani } \\
\text { with some } \\
\text { Tshakur } \\
\text { words; older } \\
\text { men also } \\
\text { Russian }\end{array}$ & $\begin{array}{l}\text { Budukh and } \\
\text { Azeri mono- } \\
\text { lingual in } \\
\text { Russian; } \\
\text { some bilin- } \\
\text { gual in Azeri } \\
\text { and Russian }\end{array}$ & $\begin{array}{l}\text { Bilingual } \\
\text { Khinalug and } \\
\text { Azeri; few } \\
\text { men fluent in } \\
\text { Russian }\end{array}$ & $\begin{array}{l}\text { Kryts and } \\
\text { Azeri, } \\
\text { elderly men } \\
\text { also Russian }\end{array}$ & $\begin{array}{l}\text { Rutul and } \\
\text { Azeri, elderly } \\
\text { and educated } \\
\text { people fluent } \\
\text { in Russian }\end{array}$ & $\begin{array}{l}\text { Tsakhur and } \\
\text { Azeri; some } \\
\text { elderly people } \\
\text { also Russian }\end{array}$ \\
\hline $\begin{array}{l}\text { Number of study } \\
\text { participants }\end{array}$ & 9 & 10 & 9 & 9 & 10 & 10 & 9 \\
\hline $\begin{array}{l}\% / \text { mean age of } \\
\text { women in the } \\
\text { sample }\end{array}$ & $56 / 38$ & $33 / 51$ & $44 / 59$ & $33 / 60$ & $30 / 45$ & $30 / 55$ & 44 / 49 \\
\hline $\begin{array}{l}\% / \text { mean age of } \\
\text { men in the } \\
\text { sample }\end{array}$ & $44 / 46$ & 67 / 61 & $56 / 40$ & $67 / 54$ & $70 / 56$ & $70 / 46$ & $56 / 50$ \\
\hline $\begin{array}{l}\text { Overall mean } \\
\text { age }\end{array}$ & 57 & 56 & 50 & 57 & 50 & 50 & 50 \\
\hline
\end{tabular}

Semi-structured interviews were conducted in Russian by the second author, sometimes with the help of a translator. Interviews lasted from 15 to 60 minutes and were followed, if possible, by a field walk with the interviewed person. The interviewees were asked to list and show gathered and consumed wild food plants including vegetables (cooked, fried or fermented) used for preparing sarma, wild fruits and other wild plants used in sweet preserves and/or liquors, and wild plants used for recreational herbal teas drunk in the food context without any medicinal purpose (sensu Soukand et al 2013) or as snacks. Unusual uses of cultivated plants as well as those that were both cultivated and wild were also recorded. For all listed plant uses, local names and details on

Original article could be obtained from: https://link.springer.com/article/10.1007/s10531-019-01835-3

Or request from the author, who will share the work gladly: renata.soukand@unive.it 
gathering and preparation were obtained.

In each study community only one or two people that were approached refused to be interviewed, mainly due to the lack of time. Interviews were conducted only with people who gave verbal informed consent, and the Code of Ethics of the International Society of Ethnobiology (ISE 2008) was followed.

While nomenclature follows The Plant List database (2018) and the Flora Europaea (Tutin et al 1964), and the family assignments are consistent with the Angiosperm Phylogeny Group (APG) IV (Stevens 2017), plants were identified via the Flora of Azerbaijan (Osgərov 2016, Grossheim 1949, Karjagin 1950-1961) which uses slightly different nomenclature. If the plant specimen was not available, the taxon was identified based on a full description of the plant and its habitat as well as the local/Azeri/Russian name given by the interviewees. When interviewees did not differentiate taxa at the species level, e.g. referred to different species of a genus with the same name, it was identified at the genus level, even if we collected plant samples for different representatives of the genus (for example Rumex, Allium and Mentha).

\section{Data analysis}

All local plant names were transcribed using the rules of Azerbaijani for the languages without an established alphabet, and Azerized Tsakhur and the Roman alphabet for the Khinalug and Rutul languages. Data was transcribed from field notebooks and classified according to taxa and use categories. Emic use categories were used and Use Instances (UI - the emic category of use of a taxon) served as a basis for comparison.

Further, we compared current UIs and taxa recorded for all the study communities to evaluate their foodethnobotanical distance using proportional Venn diagrams and Jaccard Similarity Indices (JI) following the methodology of González-Tejero et al. (2008): $\mathrm{JI}=(\mathrm{C} /(\mathrm{A}+\mathrm{B}-\mathrm{C})) \mathrm{x} 100$, where A represents the number of taxa/UI in sample $\mathrm{A}, \mathrm{B}$ is the number of taxa/UI in sample $\mathrm{B}$, and $\mathrm{C}$ is the number of taxa/UI common to $\mathrm{A}$ and $\mathrm{B}$. For visualization of results we used software developed by BioTuring Inc., San Diego California USA, www.bioturing.com.

For comparison and calculation of JI, some species were considered as one taxon (Mentha) whereas others were attributed to two: acidic (referred to as Rumex acetosa) and non-acidic (referred to as Rumex patientia). In addition, Allium spp. represented all Allium species apart from A. ursinum and A. rotundum, which could be accurately identify and thus treated as separate taxa.

\section{Results}

We recorded the food use of 65 species and 7 genera (including several possible taxa rarely differentiated at the popular level) representing 27 plant families (Table 2). The most well-represented families were Rosaceae, Asteraceae and Lamiaceae. The largest number of plants (23) were snacked on raw, yet 10 of them were snacked on only in one community and overall only a few people mentioned the use of snacks. Prepared foods dominated the list of emic food categories. Qutab (covered pie) is the most popular dish made with wild foods, for which 17 taxa were intensively used. Conceptually similar foods, grits or khinkali (type of dumplings), were prepared from six taxa. The leaves of eight taxa were used for wrapping sarma and six taxa were a component of dovğa (a yogurt soup). Eleven taxa were lactofermented in brine. Sweet preserves constituted another large group, for which 15 taxa were used to prepare sweet preserves and 10 for making kompot. Recreational tea consisted of eight taxa, while four were used only in the Azerized Tsakhur community.

Table 2. Wild food plants recorded among the studied autochthonous communities and their local culinary uses.

\section{Latin name}

Achillea arabica;

Asteraceae (AZD18)

\section{Local names}

Koymaderan $^{\top}$

\section{Parts used}

Leaves
Uses

Seasoning for qutab and girts ${ }^{T T}$ 
Sõukand, R. and Pieroni, A. 2019. Resilience in the mountains: biocultural refugia of wild food in the Greater Caucasus Range, Azerbaijan. Biodiversity and Conservation, 28 (13): 3529-3545.

Allium rotundum;

Amaryllidaceae (AZ23)

Allium spp.";

Amaryllidaceae

Allium ursinum";

Amaryllidaceae

Amaranthus retroflexus;

Amaranthaceae (GR05,

GR19)

Brassicaceae (AZ094)

Arctium tomentosum ${ }^{\#}$;

Asteraceae

Arctostaphylos uva-ursi";

Ericaceae

Berberis vulgaris";

Berberidaceae

Calepina irregularis;

Brassicaceae (AZ67)

Capsella bursa-pastoris;

Brassicaceae (AZ24)

Carum caucasicum

Apiaceae

Castanea sativa;

Fagaceae $^{\#}$

Centaurea sp. (AZ69)

Cirsium echinus (AZ57), C. macrocephalum (AZ10);

Asteraceae

Cornus mas"; Cornaceae

Corylus avellana"; Betulaceae $\begin{array}{lll}\text { Arna }^{\top} & \text { Leaves } & \text { Kata }\end{array}$

Cincilim ${ }^{\mathrm{X}}$, Jalıjas ${ }^{\mathrm{S}}$ Jut $^{\mathrm{K}}$, Leaves

Lamzü, Məsaser,

Peləi $^{\mathrm{R}}$, Sipa $^{\top}$, Şanak ${ }^{\mathrm{B}}$

Haljar $^{S, T}$, Ramzil ${ }^{R} \quad$ Leaves

Aerial parts

Boiled $^{\mathrm{SS}}$, eaten raw $^{\mathrm{K}}$ (with bread) ${ }^{\mathrm{X}}$, fremented in brine $^{\mathrm{BBB}, \mathrm{K}, \mathrm{RR}, \mathrm{SS}}$, salads ${ }^{\mathrm{BB}}$, qutab $^{\mathrm{BBB}, S \mathrm{SS}, \mathrm{TT}}$, dovğa ${ }^{\mathrm{BBB}}$

Boiled $^{R, S}$, lactofermented in brine ${ }^{R R R, S S S, T T}$, salad ${ }^{R}$

Penjar $^{T, A}$, Penje $^{R}$

Roots

Leaves

Seasoning cold dishes ${ }^{A, R}$

Lactofermented in brine ${ }^{R}$

Spring snack ${ }^{B, K K K}$

\section{Pek $^{\text {B }}$}

$\operatorname{Varans}^{\mathrm{K}}$

Fruits

Kompot $^{\mathrm{K}}$

Mirape $^{\mathrm{X}}$, Sna $^{\mathrm{S}}$, Siriş ${ }^{\mathrm{K}, \mathrm{X}} \quad$ Fruits

Kazayax ${ }^{R}$

Leaves

Added to cooked rice ${ }^{\mathrm{K}}$, juice ${ }^{\mathrm{KK}, \mathrm{XX}}$, kompot $^{\mathrm{X}}$, lactofermented in brine ${ }^{\mathrm{KK}}$, sweet preserves ${ }^{\mathrm{K}, \mathrm{S}, \mathrm{X}}$

QutabRR

Quşapəyi ${ }^{\mathrm{K}, \mathrm{X}, \mathrm{T}}, \quad$ Leaves

Qutab ${ }^{\mathrm{KKK}, \mathrm{TTT}, \mathrm{Xxx}}$

Kazaya $^{\top}$, Tere ${ }^{\mathrm{K}}$

Çamen ${ }^{\mathrm{S}}, \quad$ Kujur $^{\mathrm{X}}$, Seeds Kurah $^{\mathrm{K}, \mathrm{B}}$

Shabalıh ${ }^{\mathrm{S}}$, Tsubıl ${ }^{\mathrm{A}, \mathrm{R}} \quad$ Nuts

Milled and mixed with salt, used as seasoning for food $^{\mathrm{BBB}, \mathrm{KKK}, \mathrm{XXX}}$, sausage seasoning ${ }^{\mathrm{S}}$

Added to soup with dried meat or other foods $s^{s}$, fried $^{\mathrm{R}}$, pilaf ${ }^{\mathrm{S}}$, snacked on raw or boiled in salted water ${ }^{A, R, S}$

Qutab $^{\mathrm{R}}$

Pealed and eaten $\operatorname{raw}^{\mathrm{R}, \mathrm{S}, \mathrm{T}}$

Ace $^{\top}$, lactofermented in brine ${ }^{A A, R R R, T}$, side-dish for very fatty lamb meat ${ }^{\top}$, sweet preserves ${ }^{A A, B, R, T T, X}$, kompot $^{\mathrm{AA}, \Pi}$

Sarma ${ }^{\top T}$, phkali ${ }^{T}$

Snack ${ }^{A, R, S}$ 
Sõukand, R. and Pieroni, A. 2019. Resilience in the mountains: biocultural refugia of wild food in the Greater Caucasus Range, Azerbaijan. Biodiversity and Conservation, 28 (13): 3529-3545.

Crataegus sp,; Rosaceae

(AZD02, AZ30)

Cydonia oblonga*; Rosaceae

Daucus carota $;$; Apiaceae

Elaeagnus rhamnoides;

Elaeagnaceae (AZ68)

Filipendula vulgaris ${ }^{\#}$;

Rosaceae

Fragaria vesca $;$; Rosaceae

Heracleum trachyloma;

Apiaceae (AZ66)

Humulus lupulus;

Cannabaceae (GR15)

Inula orientalis; Asteraceae

(AZD2)

Juglans regia\#; Juglandaceae

Malus baccata (AZ51) and $M$ orientalis (AZ65); Rosaceae

Malva parviflora; Malvaceae (AZ29)
Izgil $^{S}$, Jlene $^{A}, K^{\prime}$ Kelem $^{\top}$, Fruits Kunini $^{\top}$, Yemişan ${ }^{B, T}$

$\mathrm{Cim}^{\mathrm{R}}$, Haiva $^{\mathrm{A}}, \mathrm{Šim}^{\top}$

Leaves

Terhankac $^{\mathrm{B}}$

Roots

Aktulak $^{\mathrm{K}}$, Katsi ${ }^{\mathrm{T}}$, Fruits Kilakoholo ${ }^{\mathrm{B}}$, Kotkolo ${ }^{\mathrm{B}}$

Halatai $^{\mathrm{K}}, \mathrm{Kind}^{\mathrm{X}}$

Tuber

Shoots

Hacanak $^{\mathrm{A}}$, Çiyələk ${ }^{\mathrm{K}, \mathrm{X}} \quad$ Fruits

Baldrığan $^{R, S, T}, \operatorname{Siyn}^{\top}, \quad$ Stem

Sut ${ }^{R}$

Qulançar $^{\mathrm{R}}$

Shoots

Pandərçey

Flower

pettals

$\mathrm{Goz}^{\mathrm{S}}, \mathrm{Hek}^{\top}, \mathrm{H} \mathrm{\iota ç}{ }^{\mathrm{R}}, \mathrm{H}^{\mathrm{R}}{ }^{\mathrm{R}}$, Fruits Inkxato ${ }^{A}$

$\partial c^{A, R}, \quad \quad C ̧ i r i e \varsigma^{K, B}, \quad$ Fruits

Çirməऽ ${ }^{X}$, Çursumey ${ }^{\top}$,

Çiçemaj', Misəmo $\varsigma^{x}$, Ticemay ${ }^{\mathrm{S}}$, Salax ${ }^{\mathrm{R}}$



Mentha spp; Lamiaceae [incl. Mentha $\times$ piperita, and $M$. spicata; (AZO5, AZD03)**, M. aquatica (AZ33), $M$. longifolia (AZ16, AZ27, AZ44)]

Mespilus germanica; Rosaceae (AZ55)

Morus alba**; Moraceae
Çurki ${ }^{\mathrm{K}}$, Çurtha ${ }^{\mathrm{B}}$, Çurti $^{\mathrm{K}}$, Aerial parts $\mathrm{Kac}^{\mathrm{K}}$, LahaX, Nanə ${ }^{\mathrm{A}, \mathrm{R}, \mathrm{T}}$, Nuje $^{\top}$, Reyha ${ }^{\top}$, Sidık$^{R}$, Yarpəz $^{\mathrm{S}}$

Kidək $^{B, T}$, $\quad$ kidik $^{B, K, R}$, Fruits Kuduke $^{A}$, Kumshare ${ }^{A}$

Tot $^{\top} \quad$ Fruits
Sweet preserves ${ }^{B, T T}$, kompot $^{T T}$ lactofermented in brine $^{\mathrm{SS}}$, juice ${ }^{\mathrm{SS}}$, pekmez ${ }^{\mathrm{S}}$, snack ${ }^{\mathrm{A}}$

Sarma ${ }^{\mathrm{R}, \mathrm{TT}}$

Eaten raw ${ }^{\mathrm{B}}$

Juice $^{B, K}$, snack ${ }^{B B, K K}$, sweet preserves ${ }^{B B, K K}, \Pi$

During food shortages dried, milled and added to bread $^{\mathrm{K}}$, snack $^{\mathrm{K}, \mathrm{X}}$

Qutab ${ }^{\mathrm{KK}}$

Eaten $\operatorname{raw}^{\mathrm{BB}, \mathrm{KK}, \mathrm{XX}}$, kompot $^{\mathrm{K}}$, sweet preserves ${ }^{\mathrm{A}}$

Lactofermented in brine $\mathrm{RRR}^{\mathrm{SSS}, T T}$, boiled for making drinks $^{\top T}$, sweet preserves ${ }^{\top T}$

Boiled for food ${ }^{\mathrm{R}}$

Added to cheese as a garnish ${ }^{\text {ss }}$

Snack ${ }^{A, R R T}$, sweet preserves of unripe fruits ${ }^{S S S, T T}$, pkhali $^{\text {TTT }}$

Distilled into spirits ${ }^{\top}$, fermented in brine ${ }^{R R}$, kompot $R, T$, recreational tea ${ }^{X X}$, snack ${ }^{A, B B B}, K K K, S S S, X X X$, sweet preserves $^{T, x x}$

Boiled with dried meat and onion ${ }^{\mathrm{R}}$, eaten raw $^{\mathrm{X}}$, fried with eggs ${ }^{\mathrm{A}}$, grrts $^{\mathrm{S}}$, qutab $^{\mathrm{RRR}, \mathrm{SSS}, \mathrm{T}}$, sarma ${ }^{\mathrm{A}}$

Snaked on raw by children ${ }^{B}$

Dovğ $a^{\mathrm{BB}, \mathrm{KK}, \mathrm{RR}, \pi \mathrm{T}} \mathrm{X}$, eaten raw with bread and cheese during hearding ${ }^{X}$, food seasoning ${ }^{K, X}$, fresh seasoning

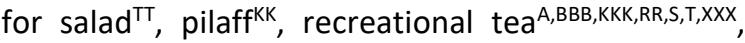
qutab ${ }^{\mathrm{BBB}, \mathrm{KKK}, \mathrm{XXX}}$

Snack ${ }^{A A, B, K}$, lactofermented in brine ${ }^{A, B B, R R R}$, juice used as seasoning for noodles ${ }^{\mathrm{B}}$, pekmez ${ }^{\mathrm{KK}, \mathrm{T}}$

Distilled into spirits ${ }^{\top}$, pekmez $^{\top}$, snack ${ }^{\top}$ 
Sõukand, R. and Pieroni, A. 2019. Resilience in the mountains: biocultural refugia of wild food in the Greater Caucasus Range, Azerbaijan. Biodiversity and Conservation, 28 (13): 3529-3545.

\begin{tabular}{|c|c|c|c|}
\hline & & Leaves & Sarma ${ }^{\top}$ \\
\hline $\begin{array}{l}\text { Oenanthe pimpinelloides; } \\
\text { Apiaceae (AZ26) }\end{array}$ & Baliarpah ${ }^{x}$ & Leaves & Mixed with cheese ${ }^{x}, q^{2} a b^{x}$ \\
\hline \multirow[t]{2}{*}{$\begin{array}{l}\text { Ornithogalum sp; } \\
\text { Asparagaceae (AZ47) }\end{array}$} & $\begin{array}{l}\text { Çag }^{\mathrm{K}} \quad \text { (bulbs), }^{\text {Çek }^{\mathrm{K}}} \text {, } \\
\text { Charghant }^{\mathrm{K}} \quad \text { (aereal }\end{array}$ & Tuber & $\begin{array}{l}\text { During food shortages was dried and milled into } \\
\text { bread or boiled }{ }^{B, K}, \text { snack }^{B B, K K, X X}\end{array}$ \\
\hline & & Leaves & Snack ${ }^{\mathrm{K}}$ \\
\hline $\begin{array}{l}\text { Papaver orientale }{ }^{\#} \text { and } P \text {. } \\
\text { rhoeas (AZ09); Papaveraceae }\end{array}$ & Parpari $^{R}$ & Seeds & Snack ${ }^{R}$ \\
\hline $\begin{array}{l}\text { Pimpinella aromatica; } \\
\text { Apiaceae (AZD05) }\end{array}$ & Yeš $^{\top}$ & Seeds & Sausage seasoning ${ }^{\top}$ \\
\hline $\begin{array}{l}\text { Plantago lanceolata; } \\
\text { Plantaginaceae (AZ61) }\end{array}$ & (Surmista) koles ${ }^{\top}$ & Leaves & $\begin{array}{l}\text { Dried and milled, added to flour when making } \\
\text { dough }{ }^{T \pi} \text {, grrts }{ }^{T} \text {, qutab }\end{array}$ \\
\hline $\begin{array}{l}\text { Plantago major; } \\
\text { Plantaginaceae (AZ22) }\end{array}$ & Bağayarpağ $\mathrm{e}^{\top, S}$, Koles $^{\top}$ & Leaves & $\begin{array}{l}\text { Dried and milled, added to flour when making } \\
\text { dough }{ }^{T \pi} \text {, girts }{ }^{\top T} \text {, qutab } \\
\text { sarma }^{S s s, \pi T} \text {, recreational tea }\end{array}$ \\
\hline
\end{tabular}

Prunus cerasifera; Rosaceae Alça ${ }^{\mathrm{S}}$, Arigk, $^{\mathrm{K}}$, Hat $^{\mathrm{R}}$, Fruits

(AZ59)

Hon $^{\top}$, quani ${ }^{A}$

Ace $^{\top}$, kompot ${ }^{\top}$, dried fruits added to soup ${ }^{\mathrm{B}, \mathrm{K}}$, lactofermented in brine ${ }^{\mathrm{A}, \mathrm{R}, \mathrm{S}}$, kompot $^{\mathrm{B}, \mathrm{K}}$, side-dish for very fatty lamb meat ${ }^{\top}$, sweet preserves ${ }^{\mathrm{BB}, \mathrm{KK}}$

Prunus cerasus"; Rosaceae

Pyrus caucasica; Rosaceae

(AZ46)

Rosa canina and Rosa spp.; Rosaceae (AZD01, AZ40, AZ63)

Rubus caucasicus; Rosaceae (AZ64)

Rubus idaeus\#; Rosaceae

Rumex acetosa (AZ54) and $R$. acetosella (AZO2);

Polygonaceae

Rumex spp.; Polygonaceae [incl. R. patientia (AZ21), $R$. obtusifolius (AZ15), R. crispus (AZ32), R. conglomeratus (AZ43)]

Sambucus ebulus; Adoxaceae (AZ58)

\section{Acibalı $^{\mathrm{S}} \quad$ Fruits \\ Armut $^{\mathrm{S}}$, Çirseher ${ }^{\mathrm{B}, \mathrm{T}}$, Fruits $\mathrm{H}_{\mathrm{I}}{ }^{\mathrm{R}}, \mathrm{Naxaxo}^{\mathrm{A}}$ \\ Çimşe ${ }^{\mathrm{k}}$, Çimke ${ }^{\mathrm{k}}$, Fruits Çimtik ${ }^{\mathrm{K}}$, Çunu $u^{\mathrm{B}, \mathrm{K}}$, Itburnu ${ }^{\mathrm{X}}$, Kaškala ${ }^{\mathrm{S}, \mathrm{T}}$, Neçep ${ }^{R}$}

Anjina ${ }^{A}$, Bəjutkan ${ }^{\mathrm{s}}$, Fruits Boguli ${ }^{\top}$, Bugürtkan ${ }^{R, X}$, Çouxul ${ }^{R}$, Kalakke $^{\mathrm{A}}$

Hura $^{S, T}$, Muxale ${ }^{A}$, Fruits Muruk $^{A}$
Haletin ${ }^{B}$, Kusuxulaga ${ }^{\mathrm{X}}$, Leaves Kuzugulag K,S, Turşag ${ }^{X}$ Mai $^{\mathrm{B}}$, Tsifu $^{\mathrm{A}}$, Tsurtsum $^{\mathrm{R}}$
Incel ${ }^{\mathrm{x}}$, Inzer ${ }^{\mathrm{x}}$, Lors ${ }^{\mathrm{K}}$, Leaves, stem Lurs ${ }^{B}$, LIS $^{\mathrm{R}}$, Kolas $^{\mathrm{S}}$, Koleç $^{R}$, Koles $^{\top}$

Sweet preserves ${ }^{\mathrm{s}}$

Distilled into spirits ${ }^{\top}$, $\operatorname{kompot}^{\top}$, snack ${ }^{A, B, S}$, sweet preserves $^{\mathrm{A}, \mathrm{T}}$

Juice ${ }^{B, K, S, X}, \quad$ kompot $^{B, K, X}$, pekmez $z^{S}$ recreational

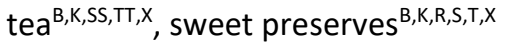

Sweet preserves ${ }^{A, R, S, T, X}$

Sweet preserves ${ }^{\mathrm{A}, \mathrm{S}, \mathrm{T}}$

$\operatorname{Dov}_{\breve{g} a^{\mathrm{XX}}}$, grits $^{\mathrm{SS}}$, qutab $^{\mathrm{A}, \mathrm{K}, \mathrm{R}, \mathrm{S}}$

Dried and milled, added to flour when making

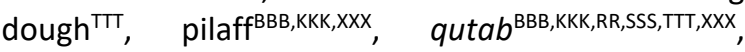
$\operatorname{dovğ} a^{\mathrm{BBB}, \mathrm{KKK}, \mathrm{XXX}}$

Fruits
Distilled into spirits ${ }^{\mathrm{A}, \mathrm{SS}, \mathrm{TT}}$, snack $^{\top}$, sweet preserves ${ }^{\top}$ 
Sõukand, R. and Pieroni, A. 2019. Resilience in the mountains: biocultural refugia of wild food in the Greater Caucasus Range, Azerbaijan. Biodiversity and Conservation, 28 (13): 3529-3545.

\begin{tabular}{|c|c|c|c|}
\hline $\begin{array}{l}\text { Sorbus caucasica; Rosaceae } \\
\text { (AZ72) }\end{array}$ & Ajalmasəs & Fruits & Snack ${ }^{S}$ \\
\hline $\begin{array}{l}\text { Stellaria media; } \\
\text { Caryophyllaceae (AZO8) }\end{array}$ & Cincilin $^{A, R, S, T, X}$ & Aerial parts & Eaten raw with bread ${ }^{\mathrm{X}}$, qutab $^{\mathrm{AAA}, \mathrm{R}, \mathrm{SS}, \mathrm{TTT}}$ \\
\hline $\begin{array}{l}\text { Thymus caucasicus (AZD08), } \\
T . \text { collinus (AZD07), and T. } \\
\text { transcaucasicus (AZ62); } \\
\text { Lamiaceae }\end{array}$ & $\begin{array}{l}\text { Çengal }{ }^{K, B}, \quad \text { Engal }^{\mathrm{B}, \mathrm{K}}, \\
\text { Kababot }^{\top}, \\
\text { Kaliglotu }^{\mathrm{B}, \mathrm{R}, \mathrm{T}, \mathrm{X}}, \quad \text { Kəkot }^{\mathrm{S}} \\
\text { Nurs }^{\mathrm{B}}\end{array}$ & Aerial parts & $\begin{array}{l}\text { Dovğ } a^{B}, \text { (added to salt mixture for) seasoning }{ }^{B, K, T T, X}, \\
\text { qutab }^{S S, X} \text {, recreational tea }{ }^{B B B, K K K, S S, T, X X X} \text {, sausage and } \\
\text { meat seasoning }{ }^{R, S S}, \text { snack }^{K}\end{array}$ \\
\hline $\begin{array}{l}\text { Tilia begoniifolia; Malvaceae } \\
\text { (GR25) }\end{array}$ & Çoka ${ }^{\mathrm{S}}, \mathrm{Hid}^{\mathrm{A}}$, Katiande ${ }^{\mathrm{A}}$ & $\begin{array}{l}\text { Leaves } \\
\text { Flowers }\end{array}$ & $\begin{array}{l}\text { Sarma (fresh and fermented) }{ }^{\mathrm{AA}, \mathrm{TT}} \\
\text { Recreational tea }^{\mathrm{S}}\end{array}$ \\
\hline $\begin{array}{l}\text { Tragopogon sp.; Asteraceae } \\
\text { [incl. T. graminifolius (AZ25) } \\
\text { and T. reticulatus (AZO3)] }\end{array}$ & $\begin{array}{l}\text { Aleminkx, }{ }^{\mathrm{x}}, \quad \text { Mhunkai }^{\mathrm{K}} \text {, } \\
\text { Yohahun }^{\mathrm{K}}\end{array}$ & leaves & Qutab ${ }^{\mathrm{K}, \mathrm{X}}$, snack $^{\mathrm{XX}}$ \\
\hline $\begin{array}{l}\text { Trifolium pratense; Fabaceae } \\
\text { (AZ73) }\end{array}$ & Yonças & Flowers & Recreational teas \\
\hline $\begin{array}{l}\text { Tussilago farfara; Asteraceae } \\
\text { (AZD12) }\end{array}$ & $\begin{array}{l}\text { Çurkik, } \\
\text { Dolmajapagartik, }{ }^{\mathrm{K}}, \text { Gaja }^{\mathrm{S}} \text {, } \\
\text { Tabilgan }{ }^{\mathrm{R}}, \quad \text { Tolpaka }^{\mathrm{K}}, \\
\text { Ugeyana }^{\mathrm{T}}\end{array}$ & leaves & $\begin{array}{l}\text { GIrts }{ }^{T} \text {, dried and milled, added to flour when making } \\
\text { dough }^{T \Pi} \text {, fermented in brine }{ }^{R} \text {, sarma }{ }^{K K, R R, S, T T}\end{array}$ \\
\hline Unidentified Asteraceae & Gelbişmek $^{\mathrm{B}}$ & leaves & Fried $^{\mathrm{B}}$, qutab $^{\mathrm{B}}$ \\
\hline Unidentified & Khermay $^{R}$ & Leaves & Qutab ${ }^{\mathrm{RR}}$ \\
\hline
\end{tabular}
root, otherwise like carrot, sweet and better than real carrot; grows on cultivated fields)

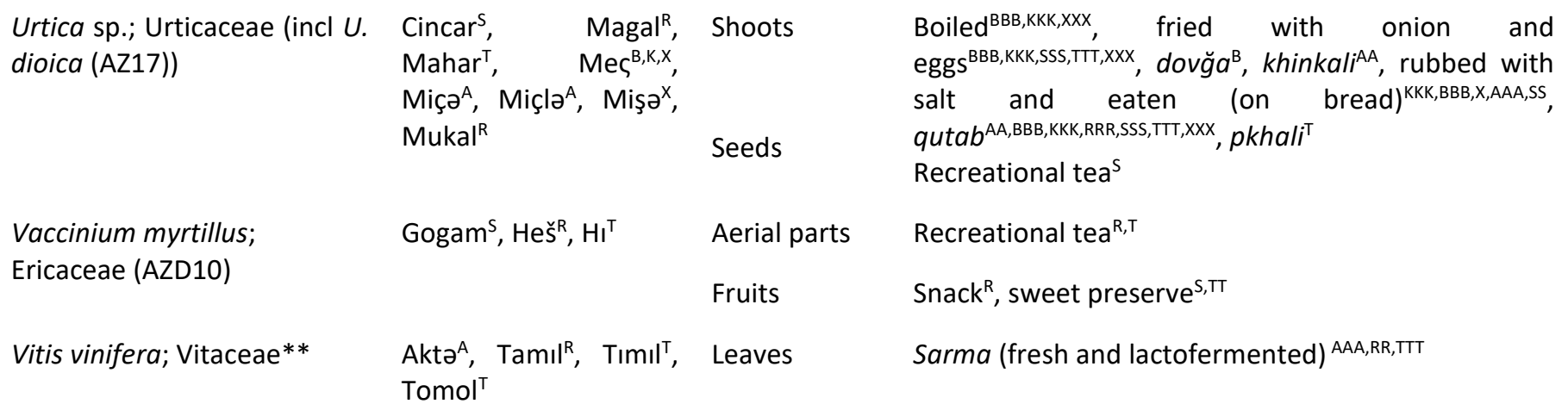

Abbreviations: Akhvakh $\left({ }^{A}\right)$, Azerized Tsakhur from Saribaş village $\left({ }^{S}\right)$, Budukh $\left({ }^{B}\right)$, Khinalug $\left({ }^{X}\right)$, Kryts $\left({ }^{K}\right)$, Rutul $\left({ }^{R}\right)$, Tsakhur $\left({ }^{T}\right)$. Gradient of use: $x-1-2$ persons, $x x-3-4$ people, $x x x-5$ or more people.

*Cultivated taxon (whose recorded folk culinary use is "unusual"); ** both wild and cultivated; \# taxon identification made through detailed plant descriptions and previosuly recorded folk names.

Dovğa - typical Azeri yogurt-and-herb based soups; gırts - Tsakhur name for khinkali, which are traditional Georgian dumplings made with various fillings (minced meat, potatoes, cheese, mushrooms and/or herbs); katə - a small open pie traditional for Tsakhurs, made

Original article could be obtained from: https://link.springer.com/article/10.1007/s10531-019-01835-3

Or request from the author, who will share the work gladly: renata.soukand@unive.it 
Sõukand, R. and Pieroni, A. 2019. Resilience in the mountains: biocultural refugia of wild food in the Greater Caucasus Range, Azerbaijan. Biodiversity and Conservation, 28 (13): 3529-3545.

of dough covered with wild garlic and salt; kompot - Slavic beverage (winter preserve) obtained by boiling fruits with a large amount of water (different from the Western European compote in the low concentration of sugar); pekmez: typical syrup of Ottoman cuisine obtained by condensing diverse fruit juices; pkhali - made from chopped greens or other vegetables mixed with ground onion, garlic, walnuts, and aromatic herbs; qutab - typical Azeri thin stuffed (salty) pancake filled with mixtures of aromatic and wild herbs, cheese, or meat.

The visual representation of wild food ethnobotanical distances (Figure 2a) demonstrates a clear distinction between the wild food plants used on the two sides of the mountain range. Calculated overlaps among the groups (Table 3) show high similarity between communities on one side of the mountain range. An overlap greater than $50 \%$ among the plants used was recorded for Khinalugs and Kryts and also the latter and Budukhs, all of which live on the north-eastern side of the range. Similar values were also observed for Tsakhurs and Azerized Tsakhurs as well as the former group and Rutuls, all residing on the south-western side of the range. Much lower values of JI for taxa were recorded between communities located on opposite sides of the mountain range for which the lowest overlap (below 20\%) was recorded between Kryts and Akhvakhs. Overlap from 20\% to 30\% was recorded between Khinalugs and both Akhvakhs and Tsakhurs as well as between Rutuls and both Kryts and Budukhs.

Table 3. Jaccard Indexes for taxa (lower left corner) and UIs (upper right corner).

\begin{tabular}{|l|l|l|l|l|l|l|l|}
\hline $\begin{array}{l}\text { JI taxa/ } \\
\text { JI UI }\end{array}$ & Akhvakh & Budukh & Kryts & Rutul & $\begin{array}{l}\text { "Azerized } \\
\text { Tsakhur" }\end{array}$ & Tsakhur & Khinalug \\
\hline Akhvakh & X & 12.7 & $\mathbf{8 . 5 7}$ & 25 & 17.91 & 14.94 & $\mathbf{9 . 5 2}$ \\
\hline Budukh & 32.25 & X & $\mathbf{4 8 . 3 3}$ & 10.67 & 16.46 & 13 & $\mathbf{3 4 . 4 3}$ \\
\hline Kryts & $\mathbf{1 9 . 4 4}$ & $\mathbf{5 3 . 8 5}$ & X & $\mathbf{8 . 6 4}$ & 15.48 & 11.32 & $\mathbf{4 0 . 3 2}$ \\
\hline Rutul & 44.44 & $\mathbf{2 8 . 9 5}$ & $\mathbf{2 7 . 5}$ & X & 22.97 & 21.74 & $\mathbf{9 . 4 6}$ \\
\hline $\begin{array}{l}\text { "Azerized } \\
\text { Tsakhur" }\end{array}$ & 42.1 & 30.77 & 29.28 & 47.62 & X & 19.8 & 16.88 \\
\hline Tsakhur & 47.5 & 33.33 & 28.89 & $\mathbf{5 5 . 8 1}$ & $\mathbf{5 3 . 3 3}$ & X & 13.27 \\
\hline Khinalug & $\mathbf{2 3 . 5 3}$ & 44.44 & $\mathbf{5 7 . 6 9}$ & 31.58 & 33.33 & $\mathbf{2 3 . 9 1}$ & X \\
\hline
\end{tabular}

The most extreme results are highlighted.

The use of taxa in emic food preparations (expressed in UIs) is more diverse and here the visual representation is not particularly informative (Figure 2b). Jaccard Indexes (Table 3) show low overlap between the recorded uses
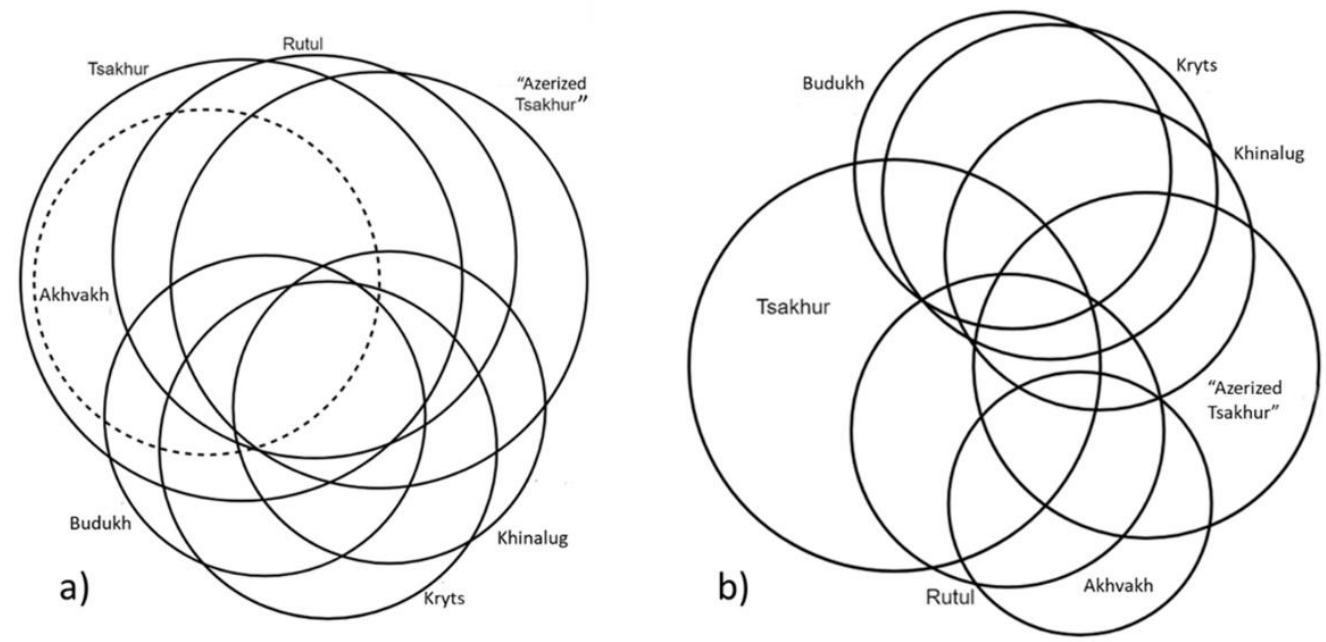

of the communities situated on opposite sides of the mountain range, being less than $10 \%$ between Kryts and both Rutuls and Akhvakhs as well as between Khinalugs and the latter two communities. Only slightly higher values (up to $13 \%$ ) were recorded for almost all other trans-range comparisons. The only exception was the 
relatively higher similarity between uses listed by Azerized Tsakhurs with all cross-range communities, ranging between $15.5 \%$ and $16.9 \%$. A high similarity of uses (from $34 \%$ to $48 \%$ ) was recorded only among communities residing on the north-eastern side of the range (NE group), while the overlap of emic uses between the communities on the south-western side of the range (SW group) remained between $15 \%$ and $25 \%$.

Figure 2. Best possible fit Venn diagram showing the overlap of taxa (a) and UI (b) for all the study communities.

Table 4. Numerical characteristics of plant use in the study communities.

\begin{tabular}{|l|l|l|l|l|l|l|l|}
\hline Parameters/communities & Akhvakh & Budukh & Kryts & Rutul & $\begin{array}{l}\text { "Azerized } \\
\text { Tsakhur" }\end{array}$ & Tsakhur & Khinalug \\
\hline Taxa used & 22 & 19 & 21 & 30 & 32 & $\mathbf{3 7}$ & $\mathbf{2 0}$ \\
\hline Unique taxa & $\mathbf{0}$ & 1 & 1 & 4 & 4 & $\mathbf{5}$ & 1 \\
\hline $\begin{array}{l}\text { Taxa used by at least 3 } \\
\text { people }\end{array}$ & $\mathbf{1 0}$ & 13 & 18 & 18 & 17 & $\mathbf{3 1}$ & 14 \\
\hline UIs & $\mathbf{2 9}$ & 42 & 47 & 41 & 50 & $\mathbf{7 1}$ & 40 \\
\hline Unique UIs & $\mathbf{4}$ & 7 & 7 & 12 & 18 & $\mathbf{2 5}$ & 10 \\
\hline $\begin{array}{l}\text { UIs named by at least 5 } \\
\text { people }\end{array}$ & $\mathbf{4}$ & $\mathbf{1 6}$ & 12 & 8 & 9 & 13 & 12 \\
\hline
\end{tabular}

The lowest and highest results for each parameter are highlighted.

The numerical characteristics of plant use (Table 4) indicate that the communities can in general be divided into two groups based on the number of taxa they mentioned: those using around 20 taxa are situated on one side of the range while those using 30 or more taxa on the other side of the range. The exception here is the Akhvakh community, which used only 22 taxa despite being located on the more "diversified" side of the range. Akhvakhs also differed in other parameters (such as an absence of culturally specific taxa, a low number of UIs and culturally significant UIs, etc.). The other noteworthy community was that of the Tsakhurs, which had the highest total in all parameters apart from UIs named by at least 5 people.

The mapping of the distribution of the use of the most important wild food taxa (named by at least three people) shows that there is only one taxon (Urtica) commonly used by all the study communities (Figure 3 ). Two more taxa were shared by six communities (Malus and non-acidic Rumex) and four (Rosa, Thymus, Mentha and nonspecified Allium) were shared by five communities. A few more plants have cross-range uses: Capsella bursapastoris was used by Khinalugs, Kryts and Tsakhurs; Budukhs and Kryts share the use of Elaeagnus rhamnoides and Prunus cerasifera with Tsakhurs and the use of Mespilus germanica with Akhvakhs; and acetic Rumex is common only among Budukhs and Azerized Tsakhurs. The remaining taxa are shared by a maximum of three communities located on the same side of the mountain range. 


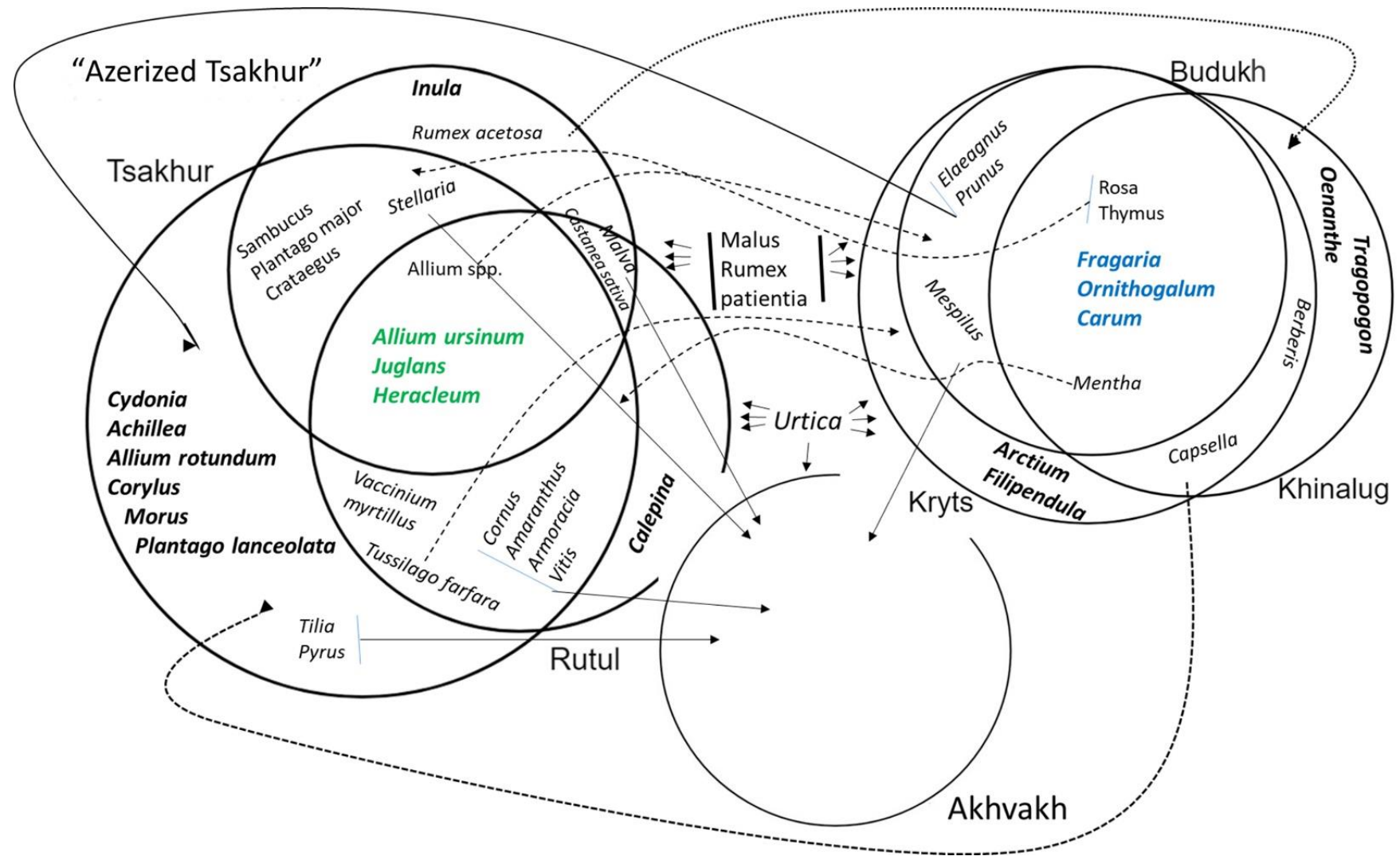

Figure 3. Distribution of the most commonly used taxa (named by at least three people) between the study communities. Highlighted are cultural markers for the study communities (bold) and regions (color).

While in the Quba region three taxa (Carum caucasicum, Fragaria vesca and Ornithogalum) are commonly used by all three communities, on the other side of the mountain not a single taxon is shared exclusively by the four communities (except for Urtica, which is equally highly used by all the study communities). The Akhvakh community clearly stands out from the others with only ten taxa commonly used and no uniquely used taxa that can be considered a cultural marker.

\section{Discussion}

Compared with other available recent research in historically or geographically close regions for which JI has been calculated, the results show remarkable differences between groups divided by mountains. For example, to date the lowest recorded level of overlap in used taxa has been between Assyrians and Muslim Kurds in Iraqi Kurdistan (32\%, Pieroni et al 2018), yet high similarity has been found between communities sharing the same side of the mountain range and language group (comparable to the overlap of closely located groups in Ukraine) (Pieroni and Soukand 2018). Although synantropic weeds are linked to horticulture, only one such taxon (Capsella bursa pastoris) is used cross-border, while the other one, Stellaria media, has been mentioned only on the south-western side of the range. With such examples and only twelve taxa commonly shared between cross-mountain communities, we can confidently say that this part of the mountain range, which has never been on the crossroads of information exchange, indeed acted as a barrier to the distribution of TEK, securing space for biocultural refugia. 


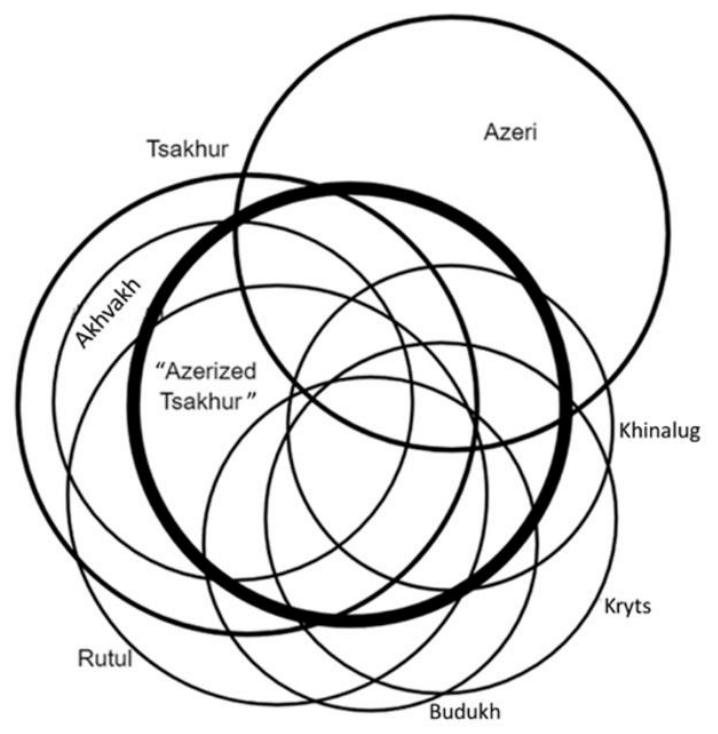

Unlike in Ukraine, where wild food has now become merely additive to everyday food or recreational tea (Pieroni and Soukand 2018), autochthonous communities in Azerbaijan keep wild plants as a main component of food (as fillings in pies, lacto-fermented side-dishes, leafy components of soups, etc.). Moreover, just two uses from times of hardship were recorded (tubers of Filipendula vulgaris and Ornitogalum sp.). Therefore, for the study communities, wild food is neither associated with food shortages nor a taste additive, but rather it is an organic part of life.

Figure 4. Best possible fit of the overlaps of taxa used by the seven study communities with the uses recorded from the Azeri population (results from Pieroni and Soukand 2019).

Azerized Tsakhurs have still kept some Tsakhur plant names (like Kaškala for Rosa) and share the largest number of taxa, but not use instances, with Tsakhurs (Table 3). At the same time, Azerized Tsakhurs also share numerous taxa with Azerbaijani communities living on the same side of the mountain range (Figure 4). This raises the question to be addressed in future research: how does assimilation by the dominant culture, in fact, work in the original ecological conditions.

The results show that the researched linguistic communities have different levels of diversity of wild food plant use and this could be related to various factors, including, but not limited to, differences in habitat surrounding the households and everyday activity spaces. However, three factors are perhaps surprising in the context of this research:

- Geographical and cultural isolation is not univocally interpretable. The community exhibiting the least diversity in wild food use (Akhvakhs) was located in close proximity to the closest town with a good access road. At the same time, two of the most isolated communities (Tsakhurs and Azerized Tsakhurs) exhibited the most diverse plant knowledge; however, the communities of Kryts and Budukh (both highly isolated) exhibited considerably lower numbers of taxa used.

- Size of the group speaking a language seems to be correlated with the diversity of plant use, with the exception of Azerized Tsakhurs who exhibited relatively high plant use diversity while having very few inhabitants in the village. This may be explained by the adaptation of Azeri food traditions alongside Azerization and the recent decrease in the number of inhabitants.

- Language as a barrier for understanding: it is possible that the linguistically northern and southern languages were also not intelligible due to limited interaction which had been for centuries the crucial factor determining marriages and exchanges of TEK. This was in place until Azeri became the lingua franca and in particular until the Soviet era when continuous contact among ethnicities became the norm.

Collective memory is supported by community interactions (Barthel et al 2013b) which can be productive only on the condition of there being a sufficient number of carriers of that memory. Depopulation is threatening such places of biocultural refugia with extinction. The few younger representatives of the communities that were interviewed were largely knowledgeable about TEK; however, interviewees in all but one community (Rutuls) stressed that young people are leaving their villages in search of jobs or education (for themselves or their 
children). Therefore, in the present context, everything that was recorded is very soon to become unlearning debt (sensu Kalle and Soukand 2016), no longer living and, more importantly, sustainable TEK.

To save biocultural refugia from sudden and evitable disappearance, forceful and immediate steps should be taken at the policy level of the country with the EU as a possible contributor. While there can be different mechanisms to promote the economic activities of certain areas, the development of regional products based on the sustainable use of local resources and unique local gastronomical knowledge could strengthen communities by providing them the economic means to continue practicing their TEK and to attain a standard of living appropriate for the $21^{\text {st }}$ century. For example, producing and marketing the distinctive cheese with petals of Inula orientalis as a local speciality could help the Azerized Tsakhurs to re-introduce pastoral activities which have almost vanished since the fall of the Soviet Union. For Tsakhurs, examples of local specialities could include the mixture of dried leaves of Rumex spp., Plantago spp. and Tussilago farfara, commonly used as an additive to pancake batter or the sweet preserves made from the stems of Heracleum trachyloma. It is important to encourage those small linguistic communities to value their TEK and to raise awareness of the importance of their TEK for the sustainability and resilience of humanity.

\section{Conclusion}

We can conclude that the majority of the researched mountain communities form distinct biocultural refugia for wild food plants, but the sustainability of such communities is now under threat due to depopulation, and their TEK has already entered the phase of unlearning debt. Therefore, proper care must be taken to encourage those communities to not only continue practicing their TEK, but also develop mechanisms to benefit from that practice through recognized regional products based on plant cultural markers. In parallel, small-scale eco-tourist activities that strongly incorporate TEK need to be developed. This, in turn, should increase the sustainability and resilience of the communities by providing on-site jobs for younger generations which would otherwise leave their community of origin. Only ensuring the transmission of the practical skills of using local plants as food and by creating favorable conditions for youth to remain or return to their villages can prevent this knowledge from dying out quickly.

Isolation is a complex mix of cultural, linguistic and natural factors and may have been responsible for the preservation of food biocultural refugia. However, at the same time, isolation may no longer be enough for the passive preservation of the food refugia in the study area into the future. More proactive steps should be taken in order to ensure the sustainability of those communities. Small-scale eco-tourist activities and small-scale city farmers' markets could reinforce a sense of identity and foster the production of local foods and herbal products. Biocultural refugia can have a future if they stop being just isolated refugia and become open refugia. Purposefully added points in a network of sustainable connections with urban and non-urban consumers will make civil societies aware of the value of biocultural diversity, increase the chance of sustainability and resilience of the existing biocultural refugia, and create a better foundation for the creation of new ones.

Aknowledgments: We are thankful to study participants who shared with authors their LEK/LGK. To the University of Gastronomic Sciences and Ca' Foscari University of Vence for having funded the field study. This research has made use of the visualization from BioVinci version 1.1.5, r20181005 developed by BioTuring Inc., San Diego California USA, www.bioturing.com.

Conflict of Interest: The authors declare that they have no conflict of interest.

Ethics: Ethical approval was granted by the Ethical Committee of the University of Gastronomic Sciences.

Herbarium data: The collected voucher specimens are deposited at the Herbarium of the Department of Environmental Sciences, Informatics, and Statistics of the Ca' Foscari University of Venice, Italy (UVV), bearing herbarium numbers UVV.EB.AZ01-73). Dried plant samples were also accepted if offered by the interviewees (deposited with numbers UVV.EB.AZD01-21). 


\section{References}

Barthel S, Crumley C, Svedin U (2013a) Bio-cultural refugia—safeguarding diversity of practices for food security and biodiversity. Global Environl Chang 23(5):1142-1152.

Barthel S, Crumley CL, Svedin U (2013b) Biocultural refugia: combating the erosion of diversity in landscapes of food production. Ecol Soc 18(4):71. Bussmann R (Ed) (2017) Ethnobotany of the Caucasus. Springer International Publishing: New York.

Bussmann RW, Zambrana NYP, Sikharulidze S, Kikvidze Z, Kikodze D, Tchelidze D, Khutsishvili M, Batsatsashvili K et al. (2016) A comparative ethnobotany of Khevsureti, Samtskhe-Javakheti, Tusheti, Svaneti, and Racha-Lechkhumi, Republic of Georgia (Sakartvelo), Caucasus. J Ethnobiol Ethnomed 12:43.

Bussmann RW, Zambrana NYP, Sikharulidze S, Kikvidze Z, Kikodze D, Tchelidze D, Khutsishvili M, Batsatsashvili et al. (2017) Plant and fungal use in Tusheti, Khevsureti, and Pshavi, Sakartvelo (Republic of Georgia), Caucasus. Acta Soc Bot Pol 86(2):3517.

Bussmann RW, Zambrana NYP, Sikharulidze S, Kikvidze Z, Kikodze D, Tchelidze D, Khutsishvili M, Batsatsashvili et al. (2018) Unequal brothers-Plant and fungal use in Guria and Racha, Sakartvelo (Republic of Georgia), Caucasus. Indian J Tradit Know 17:7-13

Osgərov A (2016) Azərbaicanin bitki aləmi. Tea Press, Baku.

Global Food Security Index (2018) https://foodsecurityindex.eiu.com/Index. Accessed 23 January 2019.

González-Tejero MR, Casares-Porcel M, Sánchez-Rojas CP, Ramiro-Gutiérrez JM, Molero-Mesa J, Pieroni A, Giustic ME, Censoriic E, et al. (2008) Medicinal Plants in the Mediterranean Area: Synthesis of the Results of the Project Rubia. J Ethnopharm 116: 341-357.

Grossheim A (1949) Opredelitel Rastenij Kavkaza. Sovetskaja Nauka, Moskva.

Hovsepyan R, Stepanyan-Gandilyan N, Melkumyan H, Harutyunyan L (2016) Food as a marker for economy and part of identity: traditional vegetal food of Yezidis and Kurds in Armenia. J Ethnic Foods 3(1): 32-41.

Kaliszewska I, Kołodziejska-Degórska I (2015) The social context of wild leafy vegetables uses in Shiri, Daghestan. J Ethnobiol Ethnomed 11:63.

Kalle R, Sõukand R (2016). Current and remembered past uses of wild food plants in Saaremaa, Estonia: Changes in the context of unlearning debt. Econ Bot 70(3):235-253.

Karjagin, I. I. (ed.) (1950-1960) Flora Azerbajdžana. AN Azerbajdžanskoj SSR Press, Baku.

Łuczaj Ł, Tvalodze B, Zalkaliani D (2017) Comfrey and Buttercup Eaters: Wild Vegetables of the Imereti Region in Western Georgia, Caucasus. Econ Bot 71(2):188-193.

Pieroni A, Nedelcheva A, Dogan Y (2015) Local knowledge of medicinal plants and wild food plants among Tatars and Romanians in Dobruja (South-East Romania). Genet Resour Crop Evol 62:605-620.

Pieroni A, Sõukand R (2019) Ethnic and religious affiliations affect traditional wild plant foraging in Central Azerbaijan. Genet Resour Crop Evol https://doi.org/10.1007/s10722-019-00802-9

Pieroni A, Sõukand R, Amin HIM, Zahir H, Kukk T (2018) Celebrating Multi-Religious Co-Existence in Central Kurdistan: the Bio-Culturally Diverse Traditional Gathering of Wild Vegetables among Yazidis, Assyrians, and Muslim Kurds. Hum Ecol 46(2):217-227.

Savo V, Salomone F, Bartoli F, Caneva G (2019) When the Local Cuisine Still Incorporates Wild Food Plants: The Unknown Traditions of the Monti Picentini Regional Park (Southern Italy). Econ Bot 73:28-46.

Sõukand R, Quave CL, Pieroni A, Pardo-de-Santayana M, Tardío J, Kalle R, Łuczaj Ł, Svanberg I, Kolosova V, AceitunoMata L, and Menendez-Baceta G (2013) Plants used for making recreational tea in Europe: a review based on specific research sites. J Ethnobiol Ethnomed 9:58.

Stevens PF (2017) Angiosperm Phylogeny Website, version 14 . Available at: http://www.mobot.org/MOBOT/research/APweb. Accessed 08.01.2019.

Stryamets N, Elbakidze M, Ceuterick M, Angelstam P, Axelsson R (2015) From economic survival to recreation: Contemporary uses of wild food and medicine in rural Sweden, Ukraine and NW Russia. J Ethnobiol Ethnomed 11:53.

The Plant List (2013) Available at: http://www.theplantlist.org/. Accessed 15.09.2017.

Tutin T, Heywood V, Burges N, Valentine D, Walters S, Webb D (1964) Flora Europaea. Cambridge University Press, Cambridge, UK. 